\title{
Participação das atividades de saúde na economia brasileira: informações da Conta de Saúde de 2000 a $2007^{1}$
}

\author{
Maria Angelica Borges dos Santos, ${ }^{2}$ Ricardo Montes de Moraes, ${ }^{3}$ \\ Tássia Gazé Holguin ${ }^{3}$ e Rebeca de La Rocque Palis ${ }^{3}$
}

Como citar Santos MAB, Moraes RM, Holguin TG, Palis RR. Participação das atividades de saúde na economia brasileira: informações da Conta de Saúde de 2000 a 2007. Rev Panam Salud Publica. 2012;31(2):153-60.

RESUMO Objetivo. Descrever a participação das atividades de saúde na economia brasileira entre 2000 e 2007 em termos de valor adicionado e geração de postos de trabalho.

Métodos. Foram utilizados dados secundários do Instituto Brasileiro de Geografia e Estatística para os anos de 2000 a 2007. Foram analisadas as seguintes atividades: saúde pública, produção de serviços de saúde e serviços sociais privados, planos de saúde, indústria farmacêutica, indústria de equipamentos médicos e comércio de produtos farmacêuticos. Foi calculada a participação de cada atividade no total da economia e no setor saúde, a participação percentual dos componentes do valor adicionado na ótica da renda para as atividades de saúde e o crescimento real do valor adicionado por atividade de saúde. Para complementar as análises, foram levantados os rendimentos médios do trabalho e o número de postos de trabalho por atividade.

Resultados. A participação do setor saúde na economia variou de 5,2 a 5,8\%. Cresceu a participação da saúde pública (de 1,7 para 2,0\%) e caiu a dos serviços de saúde privados (de 2,4 para 2,2\%). O crescimento médio anual do setor $(3,5 \%)$ foi próximo ao da economia $(3,4 \%)$. A participação do comércio de produtos farmacêuticos no setor aumentou de 9,1 para 13,2\%. As atividades com maior crescimento acumulado foram: fabricação de aparelhos médico-hospitalares $(42,7 \%)$, saúde pública $(39,4 \%)$ e planos e seguros de saúde $(35,8 \%)$. A saúde representou 4,1\% dos postos de trabalho da economia em 2000 e 4,4\% em 2007, com 1 milhão de novos postos. Os rendimentos do trabalho representaram 6,7\% do total da economia em 2000 e 7,5\% em 2007.

Conclusões. O setor saúde tem uma participação importante na economia brasileira, embora essa participação ainda seja inferior àquela observada em países de alta renda. $O$ aumento da participação dos serviços públicos no valor adicionado setorial, o crescimento das margens de comercialização dos produtos farmacêuticos e o crescimento real inferior à média setorial da indústria farmacêutica devem ser monitorados.

Palavras-chave Economia; setor de assistência à saúde; indústria farmacêutica; serviços de saúde; Brasil.

1 As opiniões, informações, dados e conceitos contidos neste trabalho são de exclusiva responsabilidade dos autores, não cabendo ao Instituto Brasileiro de Geografia e Estatística (IBGE) nenhuma responsabilidade por eles.

2 Fundação Oswaldo Cruz, Escola Nacional de Saúde Pública Sérgio Arouca, Núcleo de Tecnologia e Logística em Saúde, Rio de Janeiro (RJ), Brasil. Correspondência: Maria Angelica Borges dos Santos, angelicabsantos@ensp.fiocruz.br
Desde a década de 1950, a informação macroeconômica mais valorizada pelos países é a do gasto público e privado com bens e serviços de saúde. Tal gasto

\footnotetext{
Instituto Brasileiro de Geografia e Estatística (IBGE), Diretoria de Pesquisas, Coordenação de Contas Nacionais, Rio de Janeiro (RJ), Brasil.
}

corresponde às despesas de consumo final de saúde do governo e das famílias (1). Na última década, sentiu-se a necessidade de aprofundar as análises macroeconômicas para o setor, com acompanhamento não apenas das despesas, mas da produção de bens e serviços, 
geração de renda e participação das atividades de saúde na economia. Como pano de fundo, acirrou-se o debate entre autores que defendem o aumento da participação das atividades de saúde nas economias nacionais (2) - com consequente crescimento de geração de renda e oferta de empregos pelo setor - e os que sustentam que esse aumento de participação gera perda de competitividade no restante da economia, em função do financiamento direto ou indireto dos gastos com saúde de trabalhadores e população pelas empresas, que repercute nos custos dos produtos (3).

Vários países já começam a recomendar a elaboração de assim chamadas contas-satélites de saúde (4-6) para subsidiar a tomada de decisões sobre políticas públicas $(7,8)$ e o delineamento de estratégias empresariais para o setor (9). As contas-satélites são criadas dentro do sistema de contas nacionais (SCN), que sintetiza todas as informações econômicas de um país, inclusive o produto interno bruto (PIB). O SCN também fornece informações para a análise de setores específicos da economia, como a saúde (10). Nesse contexto, a conta-satélite permite aumentar o detalhamento e o escopo dos setores em estudo, com apresentação de quadros complementares aos divulgados para o total da economia, trazendo informações relevantes para análises setoriais específicas. Além disso, essa conta viabiliza a criação de medidas e indicadores para monitorar as atividades e produtos de saúde ao longo do tempo e realizar comparações entre países. Pode ainda ser usada como ferramenta para criar e testar modelos econômicos de avaliação de políticas públicas (6).

O Brasil adotou, em 2006, o marco metodológico de conta-satélite para sua Conta de Saúde. Atualmente, há dados para 8 anos (2000 a 2007) $(11,12)$. Uma próxima edição dessa Conta, com informações para 2008 e 2009, está prevista para 2012. Os dados da Conta de Saúde incluem, além das despesas com saúde, informações acerca da participação das atividades de saúde na economia. A participação de cada atividade na economia equivale à renda gerada a partir dessa atividade (valor adicionado).

Este trabalho descreve a participação das atividades de saúde na economia brasileira entre 2000 e 2007 com base na variação de dois indicadores, o valor adicionado e o número de postos de trabalho gerados pela atividade.

\section{MÉTODOS}

\section{Conceitos básicos}

Para produzir bens e serviços, as empresas, agrupadas em atividades econômicas, usam capital (na forma de máquinas, equipamentos e instalações) e trabalho (mão de obra) - os chamados fatores de produção. Esses dois fatores são remunerados por sua participação no processo de produção, gerando rendas que podem ser usadas por trabalhadores e proprietários de bens de capital para consumo e poupança (13).

Para o SCN, a renda gerada a partir de cada atividade produtiva equivale ao respectivo valor adicionado $(7,14)$. A participação de cada atividade na economia do país pode ser calculada dividindo-se o valor adicionado da atividade pelo valor adicionado total. $\mathrm{Na}$ ótica da renda, os componentes do valor adicionado são: as remunerações do trabalho, o rendimento de autônomos (classificado como rendimento misto, já que os autônomos desempenham simultaneamente os papéis de empresários e empregados, não sendo possível separar a remuneração do trabalho da renda do capital investido no negócio), o excedente operacional bruto (EOB) e os outros impostos, líquidos de subsídios (15).

A remuneração do trabalho abrange, principalmente, salários e contribuições sociais efetuadas em prol de empregados. A renda auferida pelo capital (na prática, pelos ativos usados no processo produtivo) está contida no EOB, que também pode ser definido como valor adicionado menos o valor de salários, contribuições sobre a folha de pagamentos e impostos sobre a atividade. O EOB é o que permanece com os proprietários após o pagamento dos insumos e da mão de obra usados na produção - é usado para remunerar os donos ou sócios das empresas, pagar dívidas e alguns tipos de imposto (como o imposto de renda) e financiar a aquisição de novos bens de capital - como máquinas e equipamentos (14). O EOB inclui também o chamado consumo de capital fixo, que equivale à depreciação dos ativos imobilizados. Esse é o motivo pelo qual atividades sem remuneração de empresários, como a saúde pública, possuem excedente operacional (15).

As variações do valor adicionado refletem o comportamento das atividades econômicas. Quando expressas em valo- res correntes, tais variações refletem simultaneamente as mudanças no volume da produção e nos preços dos produtos (15). Para dimensionar o crescimento econômico real de uma atividade, é preciso descontar as variações de preço das variações do valor adicionado, captando, assim, as variações de volume (4). A variação acumulada do volume ao longo do tempo reflete o crescimento real da atividade no período estudado.

Para complementar a análise das atividades na economia, o SCN informa o número de postos de trabalho por atividade e, dependendo das pesquisas disponíveis no país, a natureza do vínculo - com vínculo formal, sem vínculo formal ou autônomo (16). Os dados de emprego do SCN referem-se ao total de postos de trabalho ocupados, e não ao total de pessoas ocupadas em cada atividade. Para evitar a contagem desses postos de trabalho em mais de uma atividade, a metodologia do SCN contabiliza apenas os empregos diretamente gerados em cada atividade. São excluídos os empregos indiretos, como os ligados a empresas terceirizadas, que estão contabilizados nas atividades de origem dessas empresas (15).

\section{Protocolo de análise}

As atividades selecionadas para compor o setor saúde foram analisadas individualmente e segundo os quatro grupos abaixo, com as denominações originais das atividades na Conta-Satélite de Saúde brasileira listadas entre parênteses:

- saúde pública (saúde pública);

- serviços de saúde e sociais privados, que incluem a produção de serviços hospitalares privados (atendimento hospitalar), serviços extra-hospitalares privados (outras atividades relacionadas com atenção à saúde), serviços de asilos, clínicas de reabilitação e outros serviços sociais privados (serviços sociais privados) e a intermediação de planos e seguros privados de saúde (assistência médica suplementar);

- indústria de produtos de saúde, correspondendo às indústrias farmacêutica e de materiais e equipamentos médico-hospitalares (fabricação de produtos farmacêuticos e fabricação de aparelhos para uso médico-hospitalar e odontológico);

- comércio de produtos farmacêuticos e de saúde (comércio de produtos 
farmacêuticos, médicos, ortopédicos e odontológicos).

Foram calculadas: a participação de cada atividade relacionada à saúde no total da economia e no setor saúde; a participação percentual dos componentes do valor adicionado na ótica da renda para os quatro grupos de atividades de saúde acima; e as variações acumuladas de volume (crescimento real) do valor adicionado por atividade de saúde. Para complementar as análises, foram levantados os rendimentos médios do trabalho e o número de postos de trabalho ocupados por atividade.

Todas as descrições se referem ao período de 2000 a 2007. Foram utilizados dados secundários, compreendendo os resultados constantes nas duas publicações relacionadas à Conta-Satélite de Saúde brasileira do Instituto Brasileiro de Geografia e Estatística (IBGE) (11, 12). A metodologia das estimativas e os conceitos adotados foram rigorosamente compatíveis com o SCN brasileiro (17).

As séries foram harmonizadas ajustando-se os dados da publicação de 2005 a 2007 ao formato da primeira publicação. A série de 2005 a 2007 incluía duas atividades além das relacionadas para 2000 a 2005: 1) produção de serviços de saúde em hospitais universitários e em unidades de saúde do Ministério da Defesa e 2) produção de gases medicinais. A primeira série incluía também, como parte das despesas com saúde pública, as compras de medicamentos para distribuição gratuita pelo governo.
Essas compras foram realocadas como despesa específica do governo com medicamentos na série de 2005 a 2007. Portanto, para permitir a comparação com os anos de 2000 a 2004, foram excluídos os dados relativos a serviços de saúde prestados em hospitais universitários, unidades de saúde do Ministério da Defesa e a produção de gases medicinais. Também foram realizados ajustes no valor da produção da atividade saúde pública de modo a voltar a incluir as compras de medicamentos para distribuição gratuita de 2005 a 2007. Toda a produção dessa atividade foi considerada como sendo do grupo de serviços de saúde.

\section{RESULTADOS}

\section{Participação das atividades de saúde no valor adicionado}

As atividades relacionadas à saúde foram responsáveis, em média, por 5,5\% do valor adicionado total da economia brasileira no período de 2000 a 2007, com piso de 5,2\% em 2003 e teto de 5,8\% em 2007 (tabela 1). As atividades que tiveram o maior aumento de participação na economia no período foram a saúde pública e o comércio de produtos farmacêuticos, médicos, ortopédicos e odontológicos.

A participação dos serviços de saúde públicos e privados na economia permaneceu estável, mas aumentou a participação relativa da saúde pública (de 1,7 para $2,0 \%$ ) frente a uma perda de parti- cipação do grupo de atividades que compõem a produção de serviços de saúde e sociais privados (de 2,4 para 2,2\%).

As maiores participações no setor saúde (tabela 2) foram da saúde pública (média de 32,8\%) e da prestação de serviços de saúde extra-hospitalares privados (outras atividades relacionadas com atenção à saúde) (média de 23,0\%). A atividade que mais ganhou participação setorial no período foi o comércio de produtos farmacêuticos, médicos, ortopédicos e odontológicos $(9,1 \%$ do total do setor em 2000 e 13,2\% em 2007). A atividade fabricação de produtos farmacêuticos respondeu, em 2000, por 13,3\% do valor adicionado setorial, e, em 2007, por $11,7 \%$, com oscilações ao longo do período analisado.

A tabela 3 mostra os componentes do valor adicionado segundo a ótica da renda para os grupos de atividades da saúde. As remunerações representam a maior parte do valor adicionado dessas atividades: 58,5\% do total em 2000 e $65,0 \%$ em 2007. Na indústria farmacêutica, o EOB contribuiu mais para o valor adicionado do que as remunerações.

O comércio de produtos farmacêuticos, médicos, ortopédicos e odontológicos foi a atividade com maior crescimento da participação do EOB no período (de 28,2\% em 2000 para $37,6 \%$ em 2007). Nos serviços de saúde e sociais privados, houve perda das participações do EOB no período, com aumento das participações das remunerações do trabalho. O rendimento dos autônomos oscilou bastante, mas, desde 2005, parece

TABELA 1. Participação percentual do valor adicionado das atividades de saúde na economia, Brasil, 2000 a 2007

\begin{tabular}{|c|c|c|c|c|c|c|c|c|}
\hline \multirow[b]{2}{*}{ Atividade } & \multicolumn{8}{|c|}{ Valor adicionado } \\
\hline & 2000 & 2001 & 2002 & 2003 & 2004 & 2005 & 2006 & 2007 \\
\hline Total das atividades da economia (em $\mathrm{R} \$ 1000000)^{a}$ & 1021648 & 1118613 & 1273129 & 1470614 & 1666258 & 1842253 & 2034421 & 2287858 \\
\hline \multicolumn{9}{|l|}{ Participação percentual da saúde (\%) } \\
\hline Todas as atividades relacionadas à saúde & 5,7 & 5,7 & 5,5 & 5,2 & 5,3 & 5,3 & 5,6 & 5,8 \\
\hline Fabricação de produtos farmacêuticos & 0,8 & 0,7 & 0,6 & 0,6 & 0,6 & 0,7 & 0,7 & 0,7 \\
\hline $\begin{array}{l}\text { Fabricação de aparelhos para uso médico-hospitalar e } \\
\text { odontológico }\end{array}$ & 0,2 & 0,2 & 0,2 & 0,2 & 0,2 & 0,2 & 0,2 & 0,2 \\
\hline Comércio de produtos farmacêuticos, médicos, ortopédic & & & & & & & & \\
\hline e odontológicos & 0,5 & 0,5 & 0,4 & 0,5 & 0,6 & 0,6 & 0,7 & 0,8 \\
\hline Assistência médica suplementar & 0,3 & 0,5 & 0,3 & 0,2 & 0,2 & 0,2 & 0,2 & 0,3 \\
\hline Saúde pública & 1,7 & 1,7 & 1,9 & 1,8 & 1,8 & 1,8 & 1,8 & 2,0 \\
\hline Atividades de atendimento hospitalar (serviços privados) & 0,6 & 0,6 & 0,6 & 0,6 & 0,5 & 0,6 & 0,6 & 0,6 \\
\hline $\begin{array}{l}\text { Outras atividades relacionadas com atenção à saúde } \\
\text { (serviços privados) }\end{array}$ & 1,4 & 1,5 & 1,3 & 1,2 & 1,2 & 1,1 & 1,2 & 1,2 \\
\hline Serviços sociais privados & 0,1 & 0,1 & 0,1 & 0,1 & 0,1 & 0,1 & 0,1 & 0,1 \\
\hline
\end{tabular}

Fonte: Elaboração dos autores a partir de dados publicados pelo Instituto Brasileiro de Geografia e Estatística (IBGE).

a 1 US\$ = R \$ 1,95 (taxa média em 2007). 
TABELA 2. Participação percentual das atividades de saúde no valor adicionado pelo setor saúde, Brasil, 2000 a 2007

\begin{tabular}{|c|c|c|c|c|c|c|c|c|}
\hline \multirow[b]{2}{*}{ Atividade } & \multicolumn{8}{|c|}{ Participação das atividades no valor adicionado } \\
\hline & 2000 & 2001 & 2002 & 2003 & 2004 & 2005 & 2006 & 2007 \\
\hline Total das atividades relacionadas à saúde (em $R \$ 1000000$ correntes) $)^{a}$ & 57999 & 64112 & 69743 & 76940 & 88493 & 97580 & 114941 & 133525 \\
\hline $\begin{array}{l}\text { Fabricação de aparelhos para uso médico-hospitalar e odontológico } \\
\text { Comércio de produtos farmacêuticos, médicos, ortopédicos e }\end{array}$ & 3,0 & 3,1 & 3,4 & 3,6 & 3,6 & 3,8 & 3,7 & 3,5 \\
\hline odontológicos & 9,1 & 8,5 & 8,0 & 9,8 & 11,8 & 11,2 & 12,0 & 13,2 \\
\hline Atividades de atendimento hospitalar (serviços privados) & 10,3 & 10,1 & 11,1 & 10,9 & 10,2 & 11,0 & 10,7 & 10,8 \\
\hline $\begin{array}{l}\text { Outras atividades relacionadas com atenção à saúde (serviços } \\
\text { privados) }\end{array}$ & 25,4 & 26,4 & 23,8 & 23,4 & 22,5 & 20,4 & 21,6 & 20,3 \\
\hline Serviços sociais privados & 2,6 & 2,5 & 2,7 & 2,5 & 2,4 & 2,5 & 2,5 & 2,4 \\
\hline
\end{tabular}

Fonte: Elaboração dos autores a partir de dados publicados pelo Instituto Brasileiro de Geografia e Estatística (IBGE).

a 1 US\$ = R $\$ 1,95$ (taxa média em 2007).

TABELA 3. Composição do valor adicionado das atividades relacionadas à saúde pela ótica da renda segundo grupo de atividades, Brasil, 2000 a 2007

\begin{tabular}{|c|c|c|c|c|c|c|c|c|}
\hline Atividade & 2000 & 2001 & 2002 & 2003 & 2004 & 2005 & 2006 & 2007 \\
\hline \multicolumn{9}{|l|}{ Todas as atividades relacionadas à saúde } \\
\hline Valor adicionado $(\mathrm{R} \$ 1000000)^{\mathrm{a}}$ & 57999 & 64112 & 69743 & 76940 & 88493 & 97580 & 114941 & 133525 \\
\hline Remunerações (\%) & 58,5 & 58,5 & 63,8 & 65,6 & 63,8 & 65,2 & 63,5 & 65,0 \\
\hline Rendimentos de autônomos (\%) & 12,3 & 11,7 & 11,5 & 11,2 & 10,6 & 9,9 & 9,6 & 9,3 \\
\hline Excedente operacional bruto (\%) & 28,4 & 28,4 & 23,3 & 21,6 & 24,0 & 23,5 & 25,5 & 23,9 \\
\hline Outros impostos menos subsídio sobre a produção (\%) & 0,9 & 1,3 & 1,4 & 1,6 & 1,6 & 1,4 & 1,3 & 1,8 \\
\hline \multicolumn{9}{|l|}{ Saúde pública } \\
\hline Valor adicionado $(\mathrm{R} \$ 1000000)^{\mathrm{a}}$ & 17676 & 19186 & 23617 & 26554 & 30560 & 32466 & 36607 & 44816 \\
\hline Remunerações (\%) & 87,3 & 89,7 & 91,2 & 91,6 & 91,4 & 91,4 & 92,4 & 93,4 \\
\hline Rendimentos de autônomos (\%) & 0,0 & 0,0 & 0,0 & 0,0 & 0,0 & 0,0 & 0,0 & 0,0 \\
\hline Excedente operacional bruto (\%) & 12,7 & 10,3 & 8,8 & 8,4 & 8,6 & 8,6 & 7,6 & 5,6 \\
\hline Outros impostos menos subsídio sobre a produção (\%) & 0,0 & 0,0 & 0,0 & 0,0 & 0,0 & 0,0 & 0,0 & 1,0 \\
\hline \multicolumn{9}{|l|}{ Serviços de saúde privados ${ }^{b}$} \\
\hline Valor adicionado $(\mathrm{R} \$ 1000000)^{\mathrm{a}}$ & 25550 & 30063 & 30192 & 31062 & 34505 & 37490 & 45061 & 50752 \\
\hline Remunerações (\%) & 45,3 & 43,9 & 50,8 & 55,1 & 54,0 & 57,2 & 55,2 & 55,3 \\
\hline Rendimento de autônomos (\%) & 25,0 & 22,5 & 23,8 & 25,0 & 24,5 & 23,0 & 22,1 & 21,9 \\
\hline Excedente operacional bruto (\%) & 28,5 & 31,6 & 23,2 & 17,1 & 18,7 & 17,7 & 20,8 & 20,7 \\
\hline Outros impostos menos subsídio sobre a produção (\%) & 1,1 & 2,0 & 2,3 & 2,8 & 2,8 & 2,1 & 2,0 & 2,2 \\
\hline \multicolumn{9}{|l|}{ Indústrias de produtos de saúde ${ }^{c}$} \\
\hline Valor adicionado $(\mathrm{R} \$ 1000000)^{\mathrm{a}}$ & 19059 & 20028 & 21094 & 25001 & 27803 & 32979 & 36402 & 38425 \\
\hline Remunerações (\%) & 38,7 & 41,7 & 37,8 & 38,9 & 39,6 & 36,7 & 34,3 & 35,3 \\
\hline Rendimentos de autônomos (\%) & 2,7 & 2,9 & 3,0 & 2,9 & 2,8 & 2,4 & 2,2 & 2,5 \\
\hline Excedente operacional bruto (\%) & 57,2 & 53,7 & 57,5 & 56,4 & 55,9 & 59,0 & 61,8 & 60,5 \\
\hline Outros impostos menos subsídio sobre a produção (\%) & 1,4 & 1,7 & 1,7 & 1,8 & 1,8 & 1,9 & 1,6 & 1,8 \\
\hline \multicolumn{9}{|l|}{ Comércio de produtos de saúde } \\
\hline Valor adicionado $(\mathrm{R} \$ 1000000)^{\mathrm{a}}$ & 5278 & 5439 & 5595 & 7506 & 10418 & 10929 & 13841 & 17683 \\
\hline Remunerações (\%) & 61,4 & 58,3 & 67,2 & 58,9 & 45,7 & 57,3 & 55,3 & 55,1 \\
\hline Rendimentos de autônomos (\%) & 8,8 & 8,8 & 8,8 & 7,4 & 5,7 & 5,6 & 4,9 & 4,7 \\
\hline Excedente operacional bruto (\%) & 28,2 & 31,1 & 21,8 & 31,7 & 46,9 & 34,7 & 37,4 & 37,6 \\
\hline Outros impostos menos subsídio sobre a produção (\%) & 1,6 & 1,8 & 2,2 & 2,0 & 1,7 & 2,4 & 2,4 & 2,6 \\
\hline
\end{tabular}

Fonte: Elaboração dos autores a partir de dados publicados pelo Instituto Brasileiro de Geografia e Estatística (IBGE).

a 1 US\$ = R \$1,95 (taxa média em 2007).

b Inclui: atividades de atendimento hospitalar, outras atividades relacionadas com atenção à saúde, serviços sociais privados e assistência médica suplementar.

c Inclui: fabricação de produtos farmacêuticos e fabricação de aparelhos para uso médico-hospitalar e odontológico.

apresentar uma tendência descendente na composição da renda gerada nesse grupo de atividades.

A tabela 4 mostra as variações, acumuladas ano a ano, do volume do valor adicionado (crescimento real) das atividades de saúde entre 2000 e 2007.
O crescimento médio anual do valor adicionado da saúde, no período, foi de $3,5 \%$, bastante próximo aos $3,4 \%$ para o total da economia.

Mesmo tendo apresentado queda do volume em alguns anos, a atividade com maior crescimento acumulado foi a fabricação de aparelhos para uso médico-hospitalar e odontológico $(42,7 \%)$, seguida da saúde pública $(39,4 \%)$ e da assistência médica suplementar $(35,8 \%)$.

Variações acumuladas abaixo da média do setor incluíram serviços hospitalares privados (atendimento hospitalar) 
$(2,0 \%)$, serviços sociais privados $(17,1 \%)$, fabricação de produtos farmacêuticos $(18,0 \%)$ e comércio de produtos farmacêuticos, médicos, ortopédicos e odontológicos $(21,6 \%)$.

\section{Rendimentos do trabalho}

Em 2000, os rendimentos do trabalho (considerando-se as remunerações e o rendimento misto bruto) para as atividades relacionadas à saúde representaram $6,7 \%$ das remunerações e rendimentos mistos totais da economia. Essa participação cresceu no período e chegou a 7,5\% em 2007 (tabela 5).

Entre 2000 e 2007, o rendimento médio por ocupação das atividades relacionadas à saúde passou de 1,6 vez a média das atividades econômicas para 1,7 vez essa média (tabela 5 e tabela 6).

Entre 2002 e 2006, os rendimentos médios mais altos entre as atividades de saúde foram os de trabalhadores de fabricação de produtos farmacêuticos.
Em 2007, os serviços de saúde hospitalares privados (atendimento hospitalar) passaram a apresentar a maior renda média entre as atividades de saúde $(3,7$ vezes a média da economia) (tabela 5).

\section{Postos de trabalho}

A participação das atividades de saúde no total de postos de trabalho no país cresceu $30,9 \%$ no período, passando de $4,1 \%$ do total de ocupações da economia em 2000 para 4,4\% em 2007. Em torno de 1 milhão de novos postos de trabalho foram criados pela saúde no período (tabela 6).

As atividades com maior participação nas ocupações ao longo de todo o período foram a saúde pública $(32,5 \%)$, os serviços extra-hospitalares privados $(26,9 \%)$ e o comércio de produtos farmacêuticos e de saúde $(18,7 \%)$. A participação dos serviços sociais privados também foi expressiva, mas as remunerações foram baixas (tabela 6).
As atividades com maior aumento no total de ocupações foram: serviços extrahospitalares privados (outras atividades relacionadas com atenção à saúde), com 310 mil novas ocupações; saúde pública, com 286 mil; e comércio de produtos farmacêuticos, médicos, ortopédicos e odontológicos, com crescimento de 227 mil postos de trabalho (tabela 6).

\section{DISCUSSÃO}

Desde a década de 1970, são feitas no Brasil pesquisas sobre a dinâmica econômica da saúde no país (18-20). São análises independentes, com abordagens variadas às diversas atividades relacionadas à saúde, que não permitem analisar o setor com o uso de indicadores macroeconômicos rigorosamente comparáveis entre si, como neste estudo.

O setor saúde teve uma participação de $5,8 \%$ no valor adicionado em 2007 , frente a 2,2\% das atividades ligadas a refino e extração do petróleo e 3,3\% para

TABELA 4. Variação acumulada no volume do valor adicionado segundo as atividades, Brasil, 2001 a 2007

\begin{tabular}{|c|c|c|c|c|c|c|c|}
\hline \multirow[b]{2}{*}{ Atividade } & \multicolumn{7}{|c|}{ Variação acumulada no volume do valor adicionado em relação ao ano de 2000 (\%) } \\
\hline & 2001 & 2002 & 2003 & 2004 & 2005 & 2006 & 2007 \\
\hline Total da economia & 1,4 & 4,6 & 5,9 & 11,8 & 15,1 & 19,4 & 26,3 \\
\hline Atividades relacionadas à saúde & 4,1 & 6,0 & 7,0 & 10,2 & 16,7 & 21,9 & 27,6 \\
\hline Fabricação de produtos farmacêuticos & $-0,6$ & $-0,8$ & $-3,1$ & 0,3 & 12,9 & 16,1 & 18,0 \\
\hline Fabricação de aparelhos para uso médico-hospitalar e odontológico & 8,4 & 26,7 & 26,6 & 41,6 & 33,5 & 35,5 & 42,7 \\
\hline Comércio de produtos farmacêuticos, médicos, ortopédicos e odontológicos & 0,4 & $-1,3$ & $-3,0$ & 2,6 & 15,1 & 18,6 & 21,6 \\
\hline Assistência médica suplementar & 11,5 & 13,7 & 11,1 & 17,4 & 24,0 & 25,8 & 35,8 \\
\hline Saúde pública & 7,6 & 12,5 & 16,5 & 16,5 & 21,3 & 29,5 & 39,4 \\
\hline Atividades de atendimento hospitalar (serviços privados) & 4,2 & 3,2 & 3,7 & 4,8 & 5,1 & 3,2 & 2,0 \\
\hline Outras atividades relacionadas com atenção à saúde (serviços privados) & 1,8 & 1,8 & 2,1 & 7,9 & 16,1 & 23,7 & 30,2 \\
\hline Serviços sociais privados & $-0,9$ & 1,2 & 6,1 & 5,7 & 1,9 & 10,0 & 17,1 \\
\hline
\end{tabular}

Fonte: Elaboração dos autores a partir de dados publicados pelo Instituto Brasileiro de Geografia e Estatística (IBGE).

TABELA 5. Rendimento médio anual, segundo as atividades, Brasil, 2000 a 2007

\begin{tabular}{|c|c|c|c|c|c|c|c|c|}
\hline \multirow[b]{2}{*}{ Atividade } & \multicolumn{8}{|c|}{ Rendimento médio anual $(\mathrm{R} \$)^{\mathrm{a}, \mathrm{b}}$} \\
\hline & 2000 & 2001 & 2002 & 2003 & 2004 & 2005 & 2006 & 2007 \\
\hline Total da economia & 6489 & 7014 & 7478 & 8428 & 8914 & 9704 & 10551 & 11729 \\
\hline Atividades relacionadas à saúde & 10668 & 11476 & 12927 & 14358 & 14689 & 15919 & 18694 & 19912 \\
\hline Fabricação de produtos farmacêuticos & 24605 & 25932 & 26812 & 29433 & 30758 & 36395 & 39681 & 41158 \\
\hline $\begin{array}{l}\text { Fabricação de aparelhos para uso médico-hospitalar e odontológico } \\
\text { Comércio de produtos farmacêuticos, médicos, ortopédicos e }\end{array}$ & 9346 & 9722 & 10813 & 12183 & 12766 & 13394 & 15162 & 15896 \\
\hline odontológicos & 5403 & 5388 & 6013 & 6841 & 7411 & 8330 & 9002 & 10971 \\
\hline Assistência médica suplementar & 20172 & 22066 & 15648 & 16256 & 15836 & 23899 & 24327 & 25275 \\
\hline Saúde pública & 11678 & 13596 & 15813 & 17405 & 18365 & 19629 & 24470 & 25156 \\
\hline Atividades de atendimento hospitalar (serviços privados) & 17331 & 19231 & 24405 & 29210 & 30275 & 33137 & 39467 & 43700 \\
\hline Outras atividades relacionadas com atenção à saúde (serviços & & & & & & & & \\
\hline privados) & 11902 & 12310 & 13096 & 14199 & 13771 & 14551 & 16238 & 17297 \\
\hline Serviços sociais privados & 3435 & 2981 & 3765 & 4017 & 4081 & 4289 & 4971 & 5570 \\
\hline
\end{tabular}

Fonte: Elaboração dos autores a partir de dados publicados pelo Instituto Brasileiro de Geografia e Estatística (IBGE).

a Rendimento médio = (remunerações + rendimento de autônomos)/total de postos de trabalho.

b 1 US\$ = R \$1,95 (taxa média em 2007). 
TABELA 6. Total de postos de trabalho segundo as atividades, Brasil, 2000 a 2007

\begin{tabular}{|c|c|c|c|c|c|c|c|c|}
\hline \multirow[b]{2}{*}{ Atividade } & \multicolumn{8}{|c|}{ Total de postos de trabalho } \\
\hline & 2000 & 2001 & 2002 & 2003 & 2004 & 2005 & 2006 & 2007 \\
\hline Total da economia & 78972347 & 79544414 & 82629067 & 84034981 & 88252473 & 90905673 & 93246963 & 94713909 \\
\hline Atividades relacionadas à saúde & 3212363 & 3276485 & 3368795 & 3443721 & 3759171 & 3871919 & 3946206 & 4205449 \\
\hline $\begin{array}{l}\text { Fabricação de produtos farmacêuticos } \\
\text { Fabricação de aparelhos para uso médico-hospitalar }\end{array}$ & 99735 & 101303 & 95853 & 102910 & 109304 & 111774 & 110102 & 115578 \\
\hline e odontológico & 58633 & 57290 & 60485 & 64268 & 64779 & 72047 & 71958 & 77757 \\
\hline ortopédicos e odontológicos & 558781 & 558091 & 580084 & 603883 & 660503 & 681031 & 741825 & 786368 \\
\hline Assistência médica suplementar & 48730 & 54066 & 58473 & 61947 & 66811 & 63642 & 67414 & 76992 \\
\hline $\begin{array}{l}\text { Saúde pública } \\
\text { Atividades de atendimento hospitalar (serviços }\end{array}$ & 1081604 & 1046331 & 1103791 & 1136445 & 1220383 & 1271483 & 1206071 & 1367930 \\
\hline privados) & 214412 & 221255 & 219139 & 218728 & 221637 & 230376 & 249549 & 250526 \\
\hline Outras atividades relacionadas com atenção à saúde & & & & & & & & \\
\hline $\begin{array}{l}\text { (serviços privados) } \\
\text { Serviços sociais privados }\end{array}$ & $\begin{array}{l}822709 \\
327759\end{array}$ & $\begin{array}{l}862377 \\
375772\end{array}$ & $\begin{array}{l}901157 \\
349813\end{array}$ & $\begin{array}{l}911524 \\
344016\end{array}$ & $\begin{array}{r}1050407 \\
365347\end{array}$ & $\begin{array}{r}1036380 \\
405186\end{array}$ & $\begin{array}{r}1097147 \\
402140\end{array}$ & $\begin{array}{r}1133343 \\
396955\end{array}$ \\
\hline
\end{tabular}

Fonte: Elaboração dos autores a partir de dados publicados pelo Instituto Brasileiro de Geografia e Estatística (IBGE).

distribuição de eletricidade, gás, água, esgoto e limpeza urbana (17). Também gerou empregos com remunerações em geral acima da média da economia.

$\mathrm{Na}$ indústria farmacêutica, o período analisado foi concomitante à introdução dos genéricos. Caliari e Ruiz (21) indicam que, entre 2000 e 2005, houve um aumento de 4,7 para $21,7 \%$ na participação de empresas brasileiras na produção total de medicamentos no país. $\mathrm{O}$ número de empresas com mais de 1000 empregados subiu de seis para 11 (21). Essas transformações estruturais podem explicar, pelo menos em parte, a oscilação da participação setorial. Observa-se, porém, que o crescimento real acumulado da indústria farmacêutica no período ficou abaixo da média do setor saúde e da economia como um todo, ou seja, a indústria farmacêutica cresceu menos do que o setor saúde e a economia. Além disso, em 2007, o salário médio pago pela indústria farmacêutica foi, pela primeira vez, ultrapassado pelo de outra atividade de saúde, especificamente os serviços hospitalares privados. Um comportamento inverso foi verificado para a indústria de materiais e equipamentos médico-hospitalares, em que a participação setorial cresceu e o volume acumulado do valor adicionado ultrapassou o do setor e o da economia.

Nas atividades de prestação de serviços de saúde, chama especialmente a atenção o crescimento da saúde pública comparativamente aos serviços de saúde e sociais privados. Cresceram o volume acumulado do valor adicionado $(29,6 \%)$, o número de postos de trabalho $(26,5 \%)$ e as remunerações médias. Em 2000, elas eram equivalentes a 1,8 vez a média do país. Em 2007, atingiram 2,1 vezes a média. Nesse mesmo período, o número de leitos em estabelecimentos públicos aumentou 4,1\% (22).

Os serviços de saúde e sociais privados - em especial os serviços hospitalares - perderam participação no setor. Esse panorama é compatível com o que vem sendo detectado em pesquisas sobre a infraestrutura dos serviços de saúde (22), que mostram uma redução do número de leitos privados da ordem de 13,9\% entre 1999 e 2005.

Um crescimento das remunerações médias nos serviços hospitalares privados acompanhou a retração do EOB nos serviços de saúde e sociais privados. Em 2007, as remunerações dos empregados em serviços hospitalares privados ultrapassaram, em valor, as remunerações médias da tradicionalmente bem remunerada indústria farmacêutica. Isso pode estar indicando que, ainda que o número de postos de trabalho nos serviços hospitalares privados tenha crescido menos do que o número de postos de todas as outras atividades de saúde analisadas, essa atividade vem aumentando a qualificação dos profissionais que emprega, ou então que há uma disputa maior por esses profissionais.

Os significados e as implicações da retração relativa dos serviços de saúde hospitalares privados precisariam considerar outros dados. O cenário é compatível com a permanência em ambiente hospitalar apenas de tratamentos ainda sem alternativa ambulatorial e, simultaneamente, com uma possível "seleção" de organizações mais qualificadas en- tre prestadores de serviços hospitalares. Essa é uma situação a acompanhar, pois os planos e seguros de saúde cresceram mais do que os serviços de saúde privados. Dados da Agência Nacional de Saúde Suplementar (23) também mostram um crescimento no número de beneficiários de planos de saúde. Expande-se, assim, a clientela potencial dos serviços de saúde privados em um momento de retração relativa de sua participação na economia.

O comércio de produtos farmacêuticos e de saúde tem importância em função do impacto das margens de comercialização no preço final dos medicamentos e do peso de medicamentos nos gastos das famílias no Brasil. Os medicamentos para uso humano representaram $34,8 \%$ dos gastos das famílias com o consumo de bens e serviços de saúde em 2007 (12). O comércio de produtos farmacêuticos e de saúde chama a atenção pelo substancial aumento de sua participação no valor adicionado. Esse aumento parece estar relacionado ao aumento de preços relativos, pois o crescimento real acumulado dessa atividade no período foi inferior ao do setor e da economia como um todo. $\mathrm{O}$ aumento de preços do serviço de comércio, que representa o aumento da margem cobrada pelos revendedores sobre o valor dos produtos revendidos, permitiu que, mesmo com um aumento no pessoal ocupado maior do que o crescimento no volume de serviços, o peso das remunerações diminuísse e a participação do EOB no valor adicionado nessa atividade aumentasse.

Os poucos estudos identificados sobre o valor adicionado da saúde em outros 
países analisam apenas os serviços de saúde incluídos na seção Saúde e Serviços Sociais do SCN, que correspondem às atividades que agrupamos como serviços de saúde e sociais privados e saúde pública $(24,25)$. Diante do baixo grau de harmonização internacional para informações macroeconômicas ligadas à produção e renda nas metodologias de contas de saúde, esses estudos somente possibilitam comparações com grandes ressalvas.

Os serviços de saúde e sociais privados e a saúde pública - inclusive a intermediação de planos e seguros de saúde - responderam, no Brasil, em 2007, por $4,2 \%$ do valor adicionado no país. Na Conta-Satélite de Saúde chilena, que cobre somente serviços financiados pelo governo, o valor adicionado de saúde pública e Instituciones de Salud Previsional (Isapres) equivaleu a 1,5\% do PIB em 2007, mas não incluiu a participação de prestadores privados financiados por desembolso direto ou outras eventuais fontes de financiamento (24).

Um estudo recente realizado na Alemanha definiu o setor saúde como o total dos serviços de saúde e sociais acrescido das atividades de comércio varejista de medicamentos e materiais médicos. O estudo apresenta, para 2005, uma participação de 6,1\% no valor adicionado da economia daquele país (26). No Brasil, esse mesmo conjunto de atividades (somado ao comércio atacadista de medicamentos e produtos médicos) respondeu por 5,0\% do valor adicionado em 2007.

Nos países da União Europeia e nos Estados Unidos, a participação da atividade de saúde e serviços sociais no valor adicionado em 1999 foi, respectivamente, de 6,3 e 7,2\% (27). A participação dessas mesmas atividades na economia canadense (25) foi de 7,4\% em 1992, com queda para 5,7\% em 2006. A tendência de queda da participação foi mais intensa para as atividades hospitalares.

No Brasil, não houve queda de participação dos serviços de saúde como um todo. Nos serviços hospitalares privados, no entanto, o volume de serviços teve um crescimento muito abaixo da média do setor saúde. Esse dado precisa ser analisado com cautela, tendo em vista o pequeno número de anos na série brasileira e o dinamismo aparente do mercado hospitalar, com movimentos de fusões e aquisições nos anos subsequentes aos apresentados neste estudo.

A participação do valor adicionado dos serviços de saúde na economia de países desenvolvidos $(25,27)$ é superior à registrada no Brasil. Também é bem maior o número de postos de trabalho diretos como percentual dos empregos na economia. Um estudo realizado na Comunidade Europeia mostra uma participação das atividades de saúde e serviços sociais na oferta de empregos da ordem de 9,3\% (28). No trabalho de Schneider et al. (26) sobre as atividades de saúde na economia alemã, em que pese a diferença de âmbito, a participação no total de empregos foi de 10,4\%.

As limitações dos resultados apresentados incluem o tamanho da série (8 anos), que diminui a segurança da análise de tendências, e as possíveis restrições ao uso desagregado de dados gerados pelo SCN (9). Ainda assim, a significância das principais tendências indicadas pelos agregados do SCN é, em geral, clara (29), o que transforma os grandes agregados macroeconômicos em uma ferramenta útil para acompanhar as atividades econômicas como um todo - inclusive as de setores específi$\cos (7)$, como a saúde.

\section{REFERÊNCIAS}

1. Powell-Jackson T, Mills A. A review of health resource tracking in developing countries. Health Policy Plan. 2007;22(6):353-62.

2. Fogel R. Technophysio evolution and the measurement of economic growth. J Evol Econ. 2004;14(2):217-21.

3. Sood N, Ghosh A, Escarce JJ. Employer-sponsored insurance, health care cost growth, and the economic performance of US industries. Health Serv Res. 2009;44(5 Pt 1):1449-64.

4. Aizcorbe AM, Retus BA, Smith S. Toward a health care satellite health account. Surv Curr Bus. 2008;24:24-30. Disponível em: http://www.bea.gov/scb/pdf/2008/05\%20 May/0508_healthcare.pdf Acessado em 5 de outubro de 2011.

5. Organisation for Economic Co-Operation and Development. A system of health accounts. Paris: OECD; 2011.

6. UK Centre for the Measurement of Government Activity, Office for National Statistics. Proposals for satellite accounts. Londres: UK-

7. Lequelier F, Blades D. Understanding national accounts. Paris: Organisation for Economic Co-Operation and Development; 2006. CeMGA; 2008.

\section{Conclusão}

As atividades de saúde estão entre as que mais contribuem para a economia brasileira. Porém, essa participação ainda é menor do que nos países de alta renda. As atividades com maior participação setorial no período estudado foram saúde pública, serviços de saúde extra-hospitalares privados e comércio de produtos farmacêuticos e de saúde.

Situações a acompanhar no Brasil pelo impacto potencial nos gastos de governo e famílias - apontadas pelo presente estudo incluem o crescimento da participação da saúde pública e a queda relativa dos serviços privados de saúde no total do valor adicionado, o crescimento real acumulado da indústria farmacêutica abaixo da média do setor saúde e o aumento dos preços dos serviços de comercialização de produtos farmacêuticos.

A familiaridade com conceitos macroeconômicos tende a ser cada vez mais desejável para a elaboração de políticas públicas de saúde. Os dados aqui apresentados viabilizam análises relevantes sobre as atividades de saúde no Brasil no período estudado. Informações macroeconômicas como estas, acrescidas de outras, como o investimento e os insumos consumidos pelas atividades, podem contribuir para ampliar o conhecimento sobre os sistemas de saúde.

Agradecimentos. Este trabalho integra o projeto ENSP-013-LIV-10-2-10, Avanços para a Conta-Satélite de Saúde, e contou com apoio do Programa de Apoio à Pesquisa, Desenvolvimento e Inovação em Saúde Pública da Escola Nacional de Saúde Pública, Fundação Oswaldo Cruz (INOVAENSP).
8. Rannan-Eliya RP, Lorenzoni L. Guidelines for improving the comparability and availability of private health expenditures under the system of health accounts framework. Paris: Organisation for Economic Co-Operation and Development; 2010.

9. Bos F. The national accounts as a tool for analysis and policy: past, present and future. 2006. Disponível em: http://mpra.ub.unimuenchen.de/1235/. Acessado em 5 de outubro de 2011.

10. United Nations. System of national accounts 2008. Nova Iorque: UN; 2009. 
11. Instituto Brasileiro de Geografia e Estatística. Economia da saúde: uma perspectiva macroeconômica 2000-2005. Rio de Janeiro: IBGE; 2008.

12. Instituto Brasileiro de Geografia e Estatística. Conta-satélite de saúde: Brasil 2005-2007. Rio de Janeiro: IBGE; 2009.

13. Gastaldi IP. Elementos da economia política. São Paulo: Saraiva; 2005.

14. Sérouzier M. Medir la economía de los países según el sistema de cuentas nacionales. Bogotá: Alfaomega; 2003.

15. Instituto Brasileiro de Geografia e Estatística. Sistema de contas nacionais. Rio de Janeiro: IBGE; 2008. (Série Relatórios Metodológicos.)

16. Hallak Neto J, Kozovits LR, Ramos RL, Pereira SR. Trabalho e rendimentos na nova série do sistema de contas nacionais. Rev Econ Contemp. 2008;12(3):571-92.

17. Instituto Brasileiro de Geografia e Estatística. Sistema de contas nacionais - Brasil 2004/2008. Rio de Janeiro: IBGE; 2010.

18. Cordeiro H. As empresas médicas: as transformações capitalistas da prática médica. Rio de Janeiro: Graal; 1984.

19. Braga JC, De Paula SG. Saúde e previdência: estudos de política social. São Paulo: Hucitec; 1986.
20. Negri B, Di Giovanni G, orgs. Brasil: radiografia da saúde. Campinas: Unicamp; 2001.

21. Caliari T, Ruiz R. Brazilian pharmaceutical industry and generic drugs: the political intentions and the unexpected changes. Proceedings of the Opening Up Innovation: Strategy, Organization and Technology; 2010 Jun 16-18; London, UK. Londres: Imperial College London Business School; 2010.

22. Instituto Brasileiro de Geografia e Estatística. Estatísticas da saúde: assistência médicosanitária 2005. Rio de Janeiro: IBGE; 2006.

23. Agência Nacional de Saúde Suplementar. Caderno de informação da saúde suplementar: beneficiários, operadoras e planos. Rio de Janeiro: Ministério da Saúde; 2010.

24. Chile, Ministerio de Salud. Cuenta satélite de salud: Chile 2003-2007. Santiago: Ministerio de Salud; 2009.

25. Sharpe A, Bradley C, Messinger H. The measurement of output productivity in the health care sector in Canada: an overview. Ottawa: Centre for the Study of Living Standards; 2007. (CSLS research report 2007-06.)

26. Schneider M, Krauss T, Hoffman U, Köse A. Foundations, methodology and selected results of a satellite account for the German health economy, 2005. BASYS. 2010.
Disponível em: http://www.basys.de/ aktuelles/gsk-en.pdf Acessado em 5 de outubro de 2011.

27. O'Mahony M, van Ark B, eds. EU productivity and competitiveness: an industry perspective. Can Europe resume the catching-up process? Luxemburgo: Office for Official Publications of the European Communities; 2003.

28. Buchegger R, Stöger K. Health as a growth factor: a comparative analysis. Viena: Austrian Federal Ministry of Health and Women; 2003.

29. Atkinson T. Atkinson review: final report. Measurement of government output and productivity for the national accounts. Nova Iorque: Palgrave Macmillan; 2005.

Manuscrito recebido em 19 de janeiro de 2011. Aceito em versão revisada em 1 de agosto de 2011.
ABSTRACT

\section{Share of health care activities in the Brazilian economy: information on Health Accounts from 2000 to 2007}

Objective. To describe the share of health care activities in the Brazilian economy between 2000 and 2007 in terms of economic value added and creation of jobs.

Methods. Secondary data from the Brazilian Institute of Geography and Statistics (IBGE) for the years 2000 to 2007 were employed. The following health care activities were analyzed: public health, production of private health services and private social services, health insurance, the pharmaceutical industry, medical equipment manufacturing, and medical and pharmaceutical product sales. The share of each activity in the total economy and in the health care sector was calculated, as well as the percentage share of value-added components from the perspective of income for health care activities and the real growth in value added by health care activity. To complement the analysis, the average income of workers and the number of jobs per activity were established.

Results. The participation of the health care sector in the economy ranged from $5.2 \%$ to $5.8 \%$. The share of public health increased from $1.7 \%$ to $2.0 \%$, and that of private healthcare services fell from $2.4 \%$ to $2.2 \%$. The average annual growth of $3.5 \%$ for the sector was close to the $3.4 \%$ annual growth recorded for the economy. The share of medical and pharmaceutical product commerce in the sector increased from $9.1 \%$ to $13.2 \%$. The activities with the highest accumulated growth were: manufacture of medical/hospital devices $(42.7 \%)$, public health $(39.4 \%)$, and health insurance $(35.8 \%)$. Health care represented $4.1 \%$ of jobs in the economy in 2000 vs. $4.4 \%$ in 2007, with 1 million new jobs. Income from labor represented $6.7 \%$ of the total economy in 2000 and $7.5 \%$ in 2007. Conclusions. The health care sector has an important stake in the Brazilian economy, although this share is still lower than that observed in high-income countries. The rising share of public services in the sector's added value, the relative growth of medical and pharmaceutical product sales margins, and a real growth below the average for the pharmaceutical industry should be monitored.

Key words Economics; health care sector; drug industry; health services; Brazil. 